\title{
Kernos
}

Revue internationale et pluridisciplinaire de religion grecque antique

1 | 1988

Varia

\section{Pandora ou la terrifiante féminité}

\section{Pierre Lévêque}

Édition électronique
URL : http://journals.openedition.org/kernos/81

DOI : 10.4000/kernos.81

ISSN : 2034-7871

\section{Éditeur}

Centre international d'étude de la religion grecque antique

\section{Édition imprimée}

Date de publication : 1 janvier 1988

ISSN : 0776-3824

\section{Référence électronique}

Pierre Lévêque, «Pandora ou la terrifiante féminité », Kernos [En ligne], 1 | 1988, mis en ligne le 31 janvier 2011, consulté le 01 mai 2019. URL : http://journals.openedition.org/kernos/81 ; DOI : 10.4000/kernos.81 
Kernos, 1(1988), p. 49-62.

\section{PANDORA \\ OU LA TERRIFIANTE FÉMINITÉ 1}

Que l'oeuvre d'Hésiode soit une oeuvre d'homme et destinée à être récitée devant des audiences masculines n'est pas contestable et l'on en retire l'impression que la femme est un être incontrôlable, qui vide le garde-manger et rompt les membres des pauvres hommes à qui elle inspire des désirs funestes. Encore faut-il distinguer entre la Théogonie et les Travaux : si les deux oeuvres évoquent le mythe fondamental de Pandora, directement arrimé sur celui de Prométhée en pleine atmosphère titanique, les Travaux parlent aussi de la femme réelle, de celle qui partage les travaux et les jours du paysan : elle descend, au moins symboliquement, de Pandora, mais ne relève pas exactement du même imaginaire. La difficulté du problème est que l'analyse la plus formalisée de la condition de l'homme se trouve dans la Théogonie, dans la suite directe de l'épisode de Pandora. Hésiode y fait preuve, plus encore qu'ailleurs, d'une volonté ferme de distinguer les cas de figure :

1. le célibataire

2. l'homme marié

2.1. avec une bonne épouse

2.2. avec une mauvaise épouse

à telle enseigne qu'il paraît bien embrayer du mythe étiologique sur la réalité concrète de la société.

\section{Le mariage et la partenaire féminine}

Dans les Travaux, Hésiode fixe les normes du mariage, comme de toutes les activités humaines $(695 \mathrm{sq}$.). Elles sont brèves : épouse sur la trentaine une fille pubère depuis 4 ans; épouse une vierge,

1 C'est la lecture de la très belle thèse de Jean-Claude CARRIÈRE, Les mythes et les notions morales dans les Travaux et les jours (Besançon, 1987), qui a été pour moi l'occasion de relire Hésiode. Qu'il soit remercié ici de tout ce que son amitié et sa science m'ont apporté. 
«afin de lui enseigner de sages manières»; épouse de préférence une voisine. La conclusion est importante : «d'abord examine bien tout, afin de ne pas épouser de la risée pour tes voisins».

A deux reprises, le poète porte un jugement sur le mariage. Dans la Théogonie (603 sq.), il envisage d'abord le cas du célibataire. Certes il ne voit pas le pain lui manquer, mais, dans sa vieillesse, il n'a personne qui prenne soin de lui $(\gamma \eta \rho v \kappa o ́ \mu \rho \varsigma)$ et son bien est partagé entre ses collatéraux.

Pour celui qui a eu en partage le mariage, deux cas :

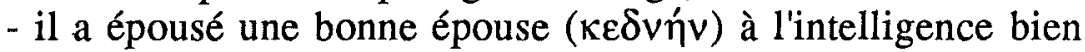
adaptée ( $\dot{\alpha} \rho \eta \rho v i \hat{\imath} \nu \pi \rho \alpha \pi i ́ \delta \varepsilon \sigma \sigma l)$ : «même ainsi il voit, toute sa vie, le mal compenser le bien»;

- il tombe sur une engeance malveillante : «alors, sa vie durant, il porte en sa poitrine un chagrin qui ne quitte plus son coeur et son mal est sans remède».

Dans les Travaux, les vers dont nous sommes parti sur l'institution du mariage sont suivis d'un jugement qui n'est point nécessairement défavorable (702 sq.) : «Il n'est pas pour l'homme de meilleure prise qu'une bonne ( $\dot{\alpha} \gamma \alpha \theta \hat{\eta} \varsigma$ ) épouse, ni en revanche

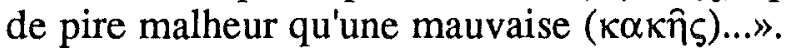

Le mariage n'est certainement pas remis en cause (comment le serait-il ?) et il est même, pour le poète, de bons mariages. Il faut toutefois reconnaître qu'Hésiode sait déployer un riche vocabulaire

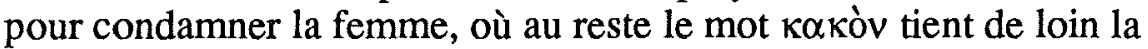
première place. Mais notons bien qu'il s'agit toujours de la fin de l'épisode de Pandore (600 sq.) :

- «la race funeste (o’ $\lambda \omega ́ 1.0 v)$ et l'engeance des femmes» (590);

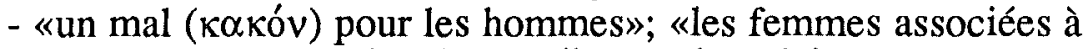

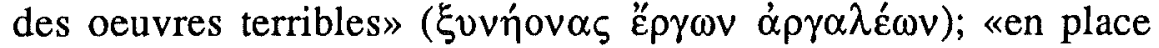

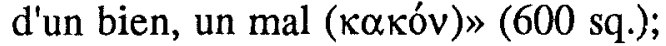

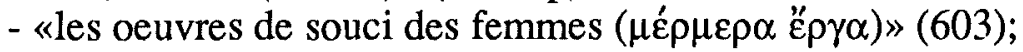

- «chagrin incessant (ou violent) et mal sans remède» (611 sq.).

Exubérance dans l'imprécation, c'est ce qui est le plus apparent. Hésiode y tient un discours manichéen - assez dans l'esprit de ses

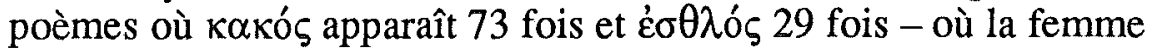

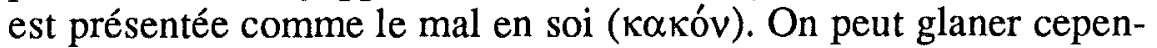


dant des indications plus précises sur les malédictions qu'elle apporte dans la vie de l'homme.

\section{La femme dévoreuse de nourriture}

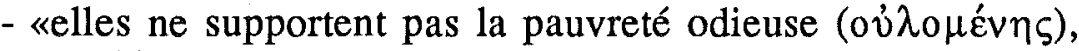
mais la satiété» (Théogonie, 593);

- «une mauvaise femme toujours à l'affut de la table

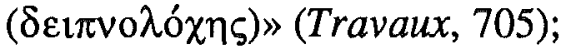

- «son babil flatteur n'en veut qu'à ta grange» (Travaux, 373).

\section{La femme inspire un désir qui ruine l'homme}

Telle Pandora (Théogonie, 66) sur qui Zeus demande à Aphrodite de répandre «le douloureux désir, les soucis qui brisent les membres», la femme est un redoutable danger, du fait des ravages qu'exerce sa féminité.

Le passage le plus clair est.dans les Travaux, 373 sq. : "Qu'une femme n'aille pas non plus, avec ses fesses attifées, te faire perdre le sens; son babil flatteur n'en veut qu'à ta grange; qui se fie à une

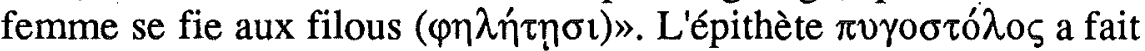
beaucoup gloser dès l'Antiquité ${ }^{2}$, mais serait-il raisonnable d'y lire autre chose qu'un art de vêtir d'une manière évocatrice ses fesses élément essentiel dans la définition de la femme ${ }^{3}$. On peut songer à ces fines tuniques de lin, épousant la forme du corps, que porteront les korés.

Toujours dans les Travaux (704 sq.), «la mauvaise épouse qui, si vigoureux que soit son mari, le consume sans torche ou le livre à

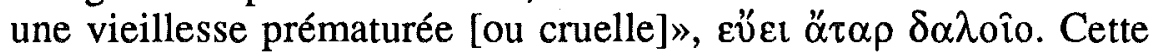
dernière menace ne peut raisonnablement renvoyer qu'à un épuisement sexuel, bien qu'on en discute allègrement.

2 Voir les scholies dans M. HOFINGER, Lexicon Hesiodeum, Leyde, 1975, s.v.

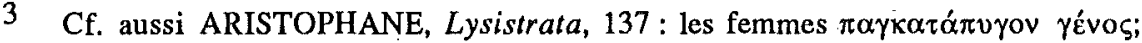
CRATINOS, fr. 53 : Katapygosynè a donné naissance à Aspasie; Katapygaina dans un graffite, Hesperia, (1953), p. 215. 
L'analyse de ces textes permet d'arriver à des conclusions claires concernant le mariage et la femme.

Hésiode n'est en rien un anarchiste et il ne remet pas en cause le" mariage qui assure la perpétuité de la famille. N'oublions ni le texte de la Théogonie (603 sq.) sur les dangers que court le célibataire, ni les vers presque touchants des Travaux sur la bonne épouse. Il y a un temps pour les noces (cf. $\dot{\omega} \rho \alpha i o \zeta$ et $̋$ pros dans Travaux, 695 et 697) et un choix attentif doit permettre de sélectionner une bonne épouse, qu'on pourra au reste former.

La femme a ses tâches, qui lui sont spécifiques. Elle enfante, mais elle élève aussi les enfants. Cela est dit expressément des filles ${ }^{4}$ en un passage d'une rare délicatesse (Travaux, 519 sq.) : «Le froid ne pénètre pas la jeune fille à la peau tendre, qui reste à l'intérieur de la maison près de sa chère mère, ignorante encore des travaux de l'Aphrodite d'or. Après avoir baigné son corps et l'avoir enduit d'huile grasse, elle va aller se coucher au fond de la maison». Il est d'autant plus nécessaire de bien élever la fille qu'elle est une richesse indispensable à l'échange des filles par pacte matrimonial entre familles voisines, sans quoi le fils ne trouverait pas à se marier.

Outre sa fonction kourutrophe, la femme travaille dans la maison. Les Travaux (779) mentionnent ses activités de tissage. Mais il faut écarter les vers fameux des Travaux (405-406) : «Ayez avant tout une maison, une femme et un boeuf de labour, une femme achetée, et non épousée, qui au besoin puisse suivre les boeufs». Ils ne concernent pas l'épouse qui oeuvre au dedans et le «avant tout» $(\pi \rho \omega \tau \iota \sigma \tau \alpha)$ indique bien les conditions nécessaires de toute exploitation rurale, même avant que le maître ne se marie sur ses trente ans.

Parfois - très rarement, et avec une extrême discrétion - est même évoqué le couple. Dans un conseil à Persès (Travaux, 399 sq.) : «Travaille..., si tu ne veux pas, un jour, avec tes enfants et ta femme, aller quêter ta vie...». Une fois même l'allusion à la sexualité de la femme est plus que claire, quand sont venus les jours pesants de l'été (Travaux, 585 sq.) : «Alors les chèvres sont plus

4 Il n'est pas question du fils, sauf dans les vers 130-131 des Travaux, équivoques puisqu'il s'agit de la race d'argent. 
grasses, le vin meilleur, les femmes plus ardentes $\left(\mu \alpha \chi \lambda{ }^{\prime} \tau \alpha \tau \alpha \imath\right)^{5}$ et les hommes plus mous». Il semble bien que ce soit un heureux temps, d'après ce qui est dit des chèvres et du vin. Il doit en être de même des chauds désirs féminins, qui ne sont pas évoqués ici comme une malédiction. La scène qui suit $(588 \mathrm{sq}$.) est une des très rares bucoliques d'Hésiode, et il me paraît probable, bien que cela ne soit pas dit, qu'elle se termine en oarystis où s'accordent l'ardeur de l'une et la mollesse de l'autre.

Alors que la cité se met en place, non sans violences contradictoires, la cellule familiale doit se renforcer sous la domination du mari/père : condition nécessaire pour que les paysans survivent, alors que «les rois mangeurs de présents» multiplient leurs excès, et surtout à un moment décisif où s'instaure une économie de plus en plus agricole, par rapport aux sociétés des Ages sombres, beaucoup plus pastorales, ainsi que l'a bien montré A. Snodgrass.

Pour que l'oikos soit stable, il faut que la femme assume ses fonctions. L'impératif majeur est donc de la bien choisir : une fille des environs, dans les 15 ans, vierge bien sûr pour qu'on puisse former cette femme/enfant - le fantasme masculin de l'Ecole des femmes est bien attesté ici au VIIIe siècle ! Mais la femme mange et veut même être rassasiée. Et c'est un être sexué, qui peut épuiser son homme, ou le tromper pour la grande joie des voisins, ou même recevoir sur sa couche son beau-frère - «répugnante faute» (Travaux, 328-329). Ces excès de la table et du lit sont de tous les temps et ils ne nous étonnent pas dans ces sociétés du haut archaïsme, où la vie est rude et les moeurs aussi. Hésiode, qui a beaucoup lutté pour la Justice, n'est guère juste en regrettant que les femmes mangent et donc coûtent, ni qu'elles suscitent des désirs qui embrasent l'homme en lui causant de terribles soucis, sans consentir à voir aucune contrepartie, alors qu'il est si apte à chanter le rôle

5 Sur la $\mu \alpha \chi \lambda$ גoóvn, on peut remarquer que le mot est employé par Hésiode dans le Catalogue (Fr. 132) à propos des filles de Proitos. Les scholiastes consta-

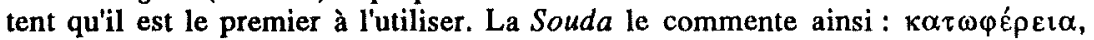

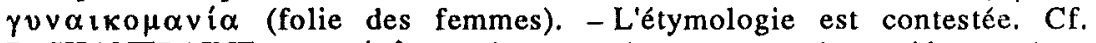

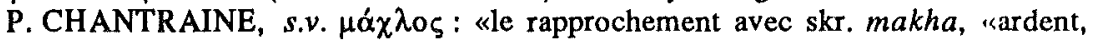
vif, gai» (?) épithète de dieux ne s'impose pas», qui n'est sans doute pas totalement convaincant. 
d'Eros, «le plus beau parmi les dieux immortels», quand il s'agit de l'économie globale du cosmos. Sa morale conjugale est un peu simple : Attention aux provisions ! Gare aux fesses attifées et au babil trompeur ! Encore ne faudrait-il pas nous laisser imposer une lecture des textes au niveau zéro et croire aux terrifiants besoins des femmes de Béotie, d'autant qu'on retrouve ici des accusations qui sont de tous les folklores campagnards. Reste que ce ton dénigreur, ces accusations traditionnelles sont un lieu de convivialité pour les hommes (Ah! les femmes...) et un moyen de les tenir en dépendance, en prétendant contrôler ce qui fait qu'elles sont elles, leur estomac et leur ventre.

Nous n'oublions pas qu'il existe d'autres passages où s'exprime un mépris radical de la femme, conçue comme mal absolu. Ils sont étonnants par l'âpreté du vocabulaire, par la vision apocalyptique qu'ils cherchent à imposer pour toute l'«engeance des femmes». D'autant que le même Hésiode termine la Théogonie par cette invocation : «Chantez, Muses Olympiennes... la race des femmes», invocation reprise au début du Catalogue où il fait revivre la gloire des héroïnes mythiques (Fr. 1, 1).

En fait, cette condamnation en bloc de la femme ne se trouve que dans les passages où elle est donnée comme l'héritière de Pandora, comme le maillon médiatique de la colère de Zeus. Ailleurs, elle est évoquée en situation hic et nunc, dans une communauté rurale localisée dans le temps et dans l'espace, et se déroule à son. sujet un discours bien plus nuancé, qui ne relève guère que d'une misogynie élémentaire. Il faut donc nous retourner vers Pandora, victime, elle, d'une misogynie radicale.

\section{Pandora : le fléau de la sexualité féminine}

Hésiode présente en Pandora un être très contrasté, mal redoutable, mais aussi vierge à l'irrésistible séduction.

Dans la Théogonie $(570,585,589)$, elle est un «mal», un «beau mal au lieu d'un bien», «un piège profond et sans issue». Mais le récit de sa création présente un être merveilleux, pareil à une chaste vierge dotée, par la volonté de Zeus, de la panoplie de la séduction : 
ceinture, robe blanche, voile brodé, diadème ciselé de bêtes innombrables.

Les Travaux apportent beaucoup plus (57 sq.). Les hommes y sont présentés avec finesse comme masochistes : «en place du feu (dit Zeus), je leur ferai présent d'un mal en qui tous, du fond du coeur, se complairont à entourer d'amour leur propre malheur»

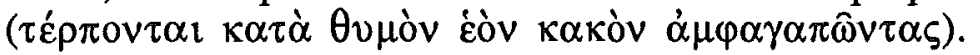

D'autre part, nous lisons deux étapes dans la genèse de Pandora : les ordres de Zeus et leur exécution. Les ordres d'abord: Athéna apprendra à Pandora «ses travaux, le métier qui tisse mille couleurs»; Aphrodite répandra sur son front «la grâce, le douloureux désir, les soucis qui brisent les membres»; Hermès mettra en elle «un esprit de chienne et un coeur artificieux».

L'exécution de ces ordres ajoute encore à ce redoutable chefd'oeuvre (72 sq.). Athéna lui noue sa ceinture et la pare. Les Charites et Peitho lui mettent des colliers d'or. Les Heures la ceignent de fleurs printanières. Athéna intervient à nouveau pour ajuster sur son corps toute sa parure, donc pour parachever les armes de la séduction. Hermès verse en son sein «mensonges, mots trompeurs, coeur artificieux» avant de lui donner la voix. Au total (83), comme dans la Théogonie, c'est un «piège profond et sans issue». Elle est toute prête à accomplir les desseins ténébreux de Zeus en séduisant Epiméthée et en ouvrant de ses mains le pithos où sont tous les maux et tous les soucis.

L'analyse de Pandora est délicate, d'autant que son ambiguïté même a entraîné des interprétations très divergentes. Mon souci n'est point de les discuter, mais de tenter de saisir la cohérence du personnage, tant en lui-même que dans une optique plus globalisante.

On ne peut échapper à l'impression que, même clairement définie par le poète comme une femme, $\gamma v \vee \alpha \hat{\imath} \kappa \alpha$, Pandora participe puissamment du monde divin.

La parure dont les dieux l'ont revêtue la prépare à l'hiérogamie. Il y a une très grande similitude de la scène avec les hiérogamies d'Héra dans l'Iliade, d'Aphrodite dans l'Hymne homérique. Le diadème d'or que lui cisèle Héphaïstos, avec ses milliers de bêtes, fait d'elle une Potnia des animaux. Certes point de mention d'union 
dans le récit, mais il est clair que tout a été fait pour qu'elle séduise Epiméthée, point faible du dispositif du frère : «il a reçu le premier la vierge formée par Zeus» (Théogonie, 513-514 sq.) et Hermès sert de médiateur en convoyant Pandora (Travaux, 83 sq.).

Pandora incarne la puissance d'attrait de la sexualité féminine, avec sa bipolarité : fraîche séduction et danger redoutable. Elle se meut ainsi dans un secteur qui est celui d'Aphrodite. Au reste, il faut relire dans la Théogonie (195 sq.) les vers où la déesse sort de l'onde : «Amour et le beau Désir lui firent cortège... Son privilège à elle, ce sont les conversations des vierges, les sourires, les tromperies, le plaisir suave, la tendresse, la douceur». Certes la tonalité de ce récit est beaucoup plus charmante : qu'on oppose par exemple «le beau désir» (Himéros) au «douloureux désir» (Pothos) et «aux soucis qui brisent les membres» (Travaux, 65 sq.). La comparaison s'impose toutefois d'autant plus que, dans les deux cas, il s'agit de la création d'un être exceptionnel, hors des normes de la procréation sexuée. Aphrodite et Pandora apparaissent bien distinctes des Mères de fécondité/fertilité dont on est tenté de les rapprocher, les deux secteurs sexualité/amour et procréation étant normalement sécants. En elles deux est un pouvoir magique, irrépressible, incontournable, qui est «le piège abrupt et sans issue» du poète.

Il ne faut pas trop opposer Aphrodite et Pandora. Les mensonges sont le lot de toutes deux et l'esprit artificieux ne peut être considéré comme totalement négatif dans une mythologie qui fait de dieux ou de héros importants les parangons d'une ruse identifiée à la sagesse. Dès le début de la Théogonie (120-122), on voit au reste Eros «qui brise les membres» tout autant que Pandora et qui, «dans la poitrine de tout dieu comme de tout homme, dompte le coeur et le sage vouloir».

Il ne faut pas non plus faire de Pandora une toute méchante créature. Elle est irresponsable, ayant été programmée pour tirer vengeance de Prométhée et des hommes bénéficiaires de ses libéralités.

Néanmoins, elle représente un pouvoir transcendant irrésistible et par là dépasse largement la condition de la femme qui est la sienne dans Hésiode. Je n'hésite pas à la rapprocher de ces Mères terribles dont je tente depuis une décennie de montrer l'importance dans la 
pensée mythíque ${ }^{6}$. Máis il faut prendre garde d'établir les distinctions nécessaires. Il y a des Mères comme Déméter la grecque ou Amaterasu la niponne qui conçoivent une épouvantable colère à la suite d'un attentat sexuel commis sur un de leurs proches ou sur elles-mêmes; elles interrompent alors le cours régulier du cosmos jusqu'à ce qu'une péripétie magique vienne changer les données et leur permette de reprendre leur personnalité de Bonnes Mères. Ce sont sans doute des déesses de ce type, blessées dans leur être le plus profond et désormais incapables de remplir leur fonction alimentaire, que représentent ees statuettes féminines au visage expressionniste, aux traits monstrueusement contractés qu'on a trouvées dans le Néolithique syro-palestinien comme à Mycènes. Rien de tel chez Pandora qui est continûment belle et qui ne subit pas de péripétie dramatique. Les Grandes Mères incarnent les ambiguités parfois crucifiantes de la reproduction alimentaire, en liaison sans doute avec le cycle végétatif; Pandora, les extrêmes dangers de la reproduction sexuée.

Personnage secondaire, quoique ici nécessaire à l'économie globale de l'univers et à une définition précise des rapports hommes/dieux après l'acte manqué de Prométhée, Pandora disparaît ensuite du récit hésiodique - sinon, nous allons le voir, de la pensée mythique -, après avoir témoigné du caractère nocif, périlleux, inquiétant de la sexualité féminine. C'est là une tendance essentielle de l'imaginaire hellénique, même si elle est tempérée par une croyance profonde dans le salut par les Mères de l'élan vital.

La Mélampodie (Fr. 275, 2) rapportait que, selon le devin Tirésias, qui avait été homme et femme, et pouvait donc comparer les deux conditions, l'homme jouissait dix fois moins que la femme. Cette peur des mystères de la féminité, parfois même du sexe féminin, est partout dans le vécu des Grecs, notamment dans leur vécu religieux. Déméter elle-même n'a pas échappé à ces suspicions. Dans les mystères, elle est qualifiée de Brimo au moment solennel où vient de naître l'enfant divin. Cette épithète signifie «la Redoutable, la Terrible, la Grondante» et Hésychius l'explique en invoquant «les

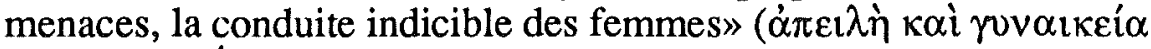
$\dot{\alpha} \rho \rho \eta \tau 0 \pi \circ$ tí $\alpha) \ldots$

6 Cf., en dernier lieu, mon livre Colère, sexe, rite. Les mythes du Japon ancien, Paris, Les Belles Lettres, 1988. 
Notons au passage que ces remarques sont loin d'épuiser le problème des Mères ou des Vierges terribles (au nombre desquelles il faudrait étudier spécialement les cas d'Héra et d'Artémis). Ni celui des Pères la Colère, dont on voit un magnifique exemple dans Hésiode avec le Zeus adversaire courroucé de Prométhée : il s'agit ici clairement d'une question de souveraineté.

\section{Pandora la polymorphe}

Bien des indices - on a dépensé des trésors de science pour les déclarer non signifiants - laissent entendre que la Pandora hésiodique n'est pas qu'une femme-robot créée à des fins spécifiques ${ }^{7}$.

Hésiode (Travaux, 80 sq.) fait violence à son nom qui ne peut guère signifier que «celle qui donne tout» ${ }^{8}$, épithète naturelle d'une Mère de fécondité/fertilité. Les témoignages ne manquent pas d'une Gè Pandora (notamment Vita herodotea Homeri, 18), d'une Rhéa Pandora (Diodore, III, 57, 2) - Rhéa représentant une fécondité sans limite qui se heurte au refus dévorateur du père Cronos. Athènes connaît une Pandora chthonienne à qui l'on sacrifie, et un cratère de l'Ashmolean Museum $\left(A R V^{2}, 1562, \mathrm{n}^{\circ} 4\right)$ représente, comme en font foi les inscriptions, Zeus, Hermès, Epiméthée au maillet et un Eros en vol au-dessus d'une Pandora qui sort du sol. Sur la base de la Parthénos était sculptée la «naissance de Pandora» (comme une anodos ?) et Pausanias, qui donne cette indication (I, 24, 7), fait référence au texte d'Hésiode : un tel emplacement, le socle de la plus célèbre statue d'Athènes, est certainement signifiant de l'importance du mythe hésiodique dans la conscience athénienne classique, d'autant qu'il est porté à la scène au moins par Sophocle. Mais il se complexise, à la fois dans le sens d'une anodos chthonienne - donc d'un retour à une Terre-Mère universelle liée aux rythmes de la vie - et dans celui d'une intégration au sein des légendes autochtones de l'Attique (la Souda, s.v. Parthénoi, fait d'elle une des six filles d'Erechthée).

7 J'ai utilisé le dossier établi par J.-Cl. CARRIÈrRE, op. cit., 2, p. 657 sq.

8 Cf. aussi le nom de l'Océanide Polydora dans la Théogonie, 354. 


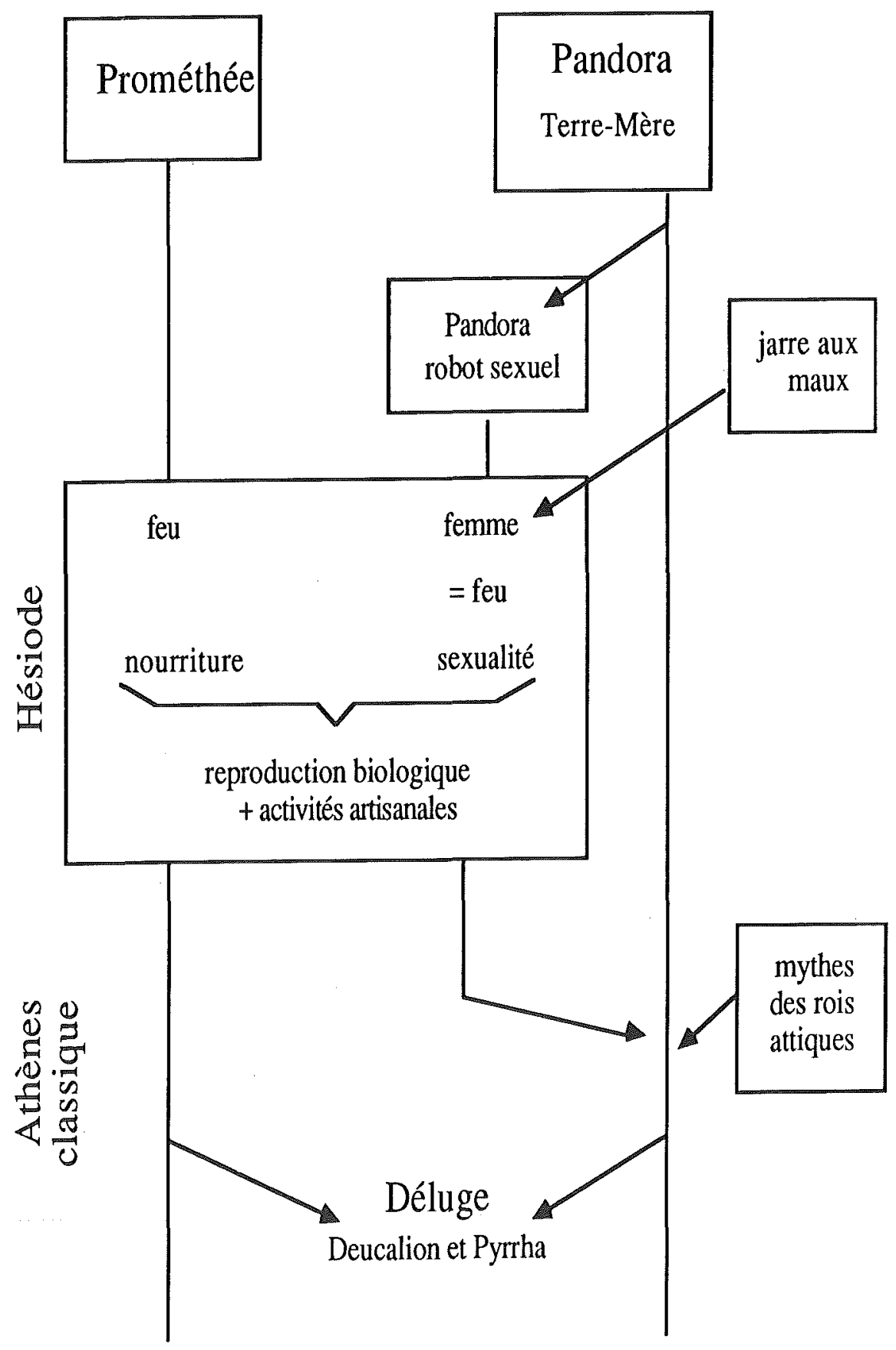


Il y a donc tout un courant (très ancien, je le répète, en raison du détournement du sens de son nom dans Hésiode) qui fait de Pandora une divinité de la Terre ou une épiclèse d'une telle divinité. Les documents athéniens témoignent de sa vitalité et Hésychius peut écrire l'équation: $\Pi \alpha v \delta \omega ́ \rho \alpha \cdot \dot{\eta} \gamma \hat{\eta}$. Mais la Pandora hésiodique n'est pas chthonienne : elle «transforme» la Terre pour jouer un rôle dans un scénario complexe, plein de suspense : la lutte de deux super-rusés, tous deux fils de Titans, Prométhée et Zeus. La Mère donatrice universelle se mue donc en une déesse/femme de l'attrait sexuel : spécification étroite, mais loin d'être arbitraire.

Cette «transformation» s'opère grâce à deux schèmes mythiques bien connus. Le premier est celui des opérations de parure nécessaires à la préparation d'une hiérogamie que j'appellerais de type Héra ou Aphrodite, c'est-à-dire d'une séquence destinée à réduire à quia, par les voies du désir amoureux, un dieu/homme peu futé. Le second est celui de la naissance exceptionnelle d'une puissance féminine qui ne sort pas du ventre d'une femme (c'est le cas d'Aphrodite et partiellement d'Athéna) et que les dieux (dans le folklore, les fées et les sorcières) dotent du nécessaire pour qu'elle affirme sa personnalité dans tel ou tel cas.

Pandora est donc, sur un point, directement comparable à Héra, Déesse-Mère chez qui la séduction n'est qu'un attrait secondaire. Elle l'est davantage encore à Aphrodite, qu'Hésiode ne considère que comme une déesse de la reproduction médiatisant les unions sexuelles (avec souvent l'expression $\delta i \dot{\alpha} \chi \rho \nu \sigma \varepsilon ́ \eta v$ 'A $\varphi \rho \circ \delta i \tau \eta \nu$, «par l'entremise de l'Aphrodite d'or»). Elle est en bonne compagnie, auprès de Dames de fécondité/fertilité, dont au moins l'une s'est aussi spécialisée dans l'amour/désir. S'il y a cependant une incontestable réduction, c'est qu'Hésiode, pour l'équilibre de son récit, ne veut voir en elle que les aspects négatifs.

Le poète semble au reste séduit par ce mythe de Pandora auquel il donne une place exceptionnelle dans la présentation des Titans (Théogonie) et quasiment au début des Travaux, où il est immédiatement suivi du mythe des races. C'est qu'il est fasciné par les grandes forces brutes que représentent les Titans et par les luttes de leurs descendants, et que Pandora s'enchasse élégamment dans le 
duel de ruses entre Zeus et Prométhée. C'est aussi qu'il est passionné de reconstruire l'histoire du monde en grandes périodes fortement tranchées et que Pandora, dans son action dégradante, introduit à la dernière étape de l'anthropogonie : les hommes accablés de tous les maux (sans qu'il y ait au reste une bonne suture avec la race de fer, non moins malheureuse).

Dans l'aventure passionnante que raconte Hésiode, il prête à Zeus des desseins d'une rare sophistication.

- Prométhée donne aux hommes le feu (ou le leur redonne sans les restrictions dont ils souffraient auparavant?). Du feu, il nous est seulement dit qu'il fait vivre (ßíov des Travaux, 42; cf. aussi 47), ce qui signifie sans doute - sens minimal - qu'il permet de cuire les aliments;

- Zeus donne aux hommes Pandora, doublement malfaisante. D'abord, elle ouvre le pithos des maux (tout en retenant l'Espérance). Ensuite elle est l'ancêtre des femmes, fléau des hommes.

Mais que d'inutiles complications dans ces desseins ténébreux! Fallait-il tant de détours à Zeus - y compris une intervention d'Hermès auprès d'Epiméthée l'imbécile - pour faire procéder à l'ouverture de la jarre aux maux, qui n'a aucun rapport visible avec Pandora? On a l'impression que l'épisode du pithos est une inclusion dans le mythe de Pandora.

Si Hésiode attache autant d'importance à cet ensemble, c'est certainement qu'il jouit intensément de ces mythes pathétiques, de ces combats de ruse qui sont l'une des premières formes de l'agon dans ce monde divin si peu fair play. Mais il doit y avoir quelque rationalité profonde dans. les ébats de ces protagonistes. Nous sommes projetés dans ces époques immémoriales où se fixait définitivement l'ordre du monde, au temps des «premiers inventeurs», en plein milieu des grands questionnements contemporains d'Hésiode. Prométhée donne le feu, donc permet aux hommes de se nourrir; Zeus donne Pandora le fléau, mais qui permet aux hommes de se reproduire et garde sans doute quelques puissances de la Terre-Mère qu'elle est aussi. Et Pandora est également un feu, puisque la femme «consume sans torche son mari» (Travaux, 705). Il semble ainsi y avoir un dénominateur commun, le feu, à entendre alors au sens le plus large, la force de la vie. Malgré les malheurs de 
ces pauvres hommes, sont donc réunies toutes les conditions nécessaires à leur reproduction biologique. Et il n'est pas exclu que les activités artisanales soient aussi impliquées: le tissage est expressément mentionné pour Pandora et les arts du feu peuvent être implicitement suggérés pour Prométhée. Tout l'avenir de la race humaine est engagé dans ces présents ambigus.

Hésiode appuie beaucoup sur la négativité du personnage de Pandora. Il a cependant montré la fin des punitions de Prométhée (Théogonie, 326 sq.) et il est possible que Pandora ait été elle aussi l'objet de quelque pardon avec retour sur ses puissances telluriques : c'est ainsi qu'on la voit à Athènes...

En tout cas, Pandora a continué à fonctionner avec Prométhée dans l'imaginaire grec. Les traditions relatives au déluge (Apollodore, I, 46) font du couple humain sauvé/salvateur Deucalion et Pyrrha les enfants de Prométhée et d'une Océanide pour lui, d'Epiméthée et de Pandora pour elle. Belle transformation où l'eau remplace le feu et où Pandora implique le salut de l'humanité.

Université de Franche-Comté

Pierre LÉVÊQUE

Faculté des Lettres

Rue Megevand 30

F - 25030 BESANÇON Cedex 